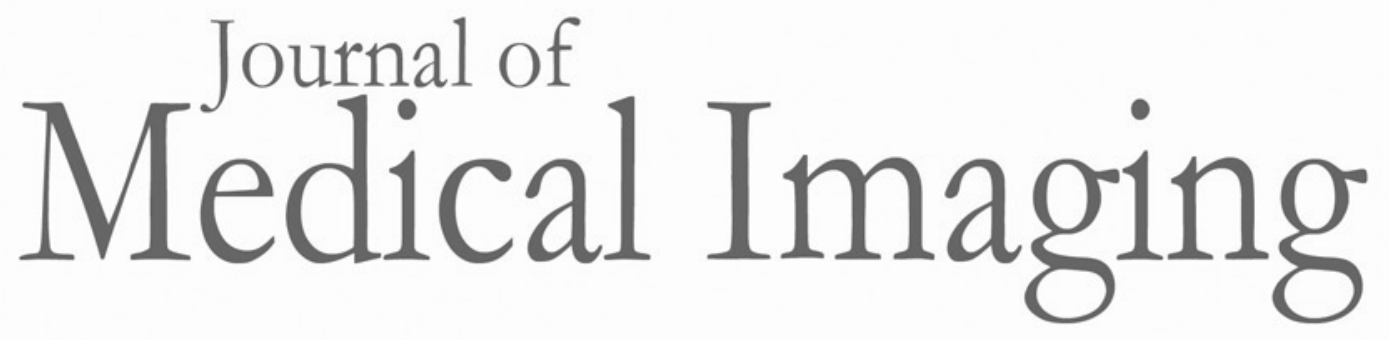

\title{
Water-fat magnetic resonance imaging quantifies relative proportions of brown and white adipose tissues: ex-vivo experiments
}

Jadranka Stojanovska

Carey N. Lumeng

Cameron Griffin

Diego Hernando

Udo Hoffmann

Jonathan W. Haft

Karen M. Kim

Charles F. Burant

Kanakadurga Singer

Alex Tsodikov

Benjamin D. Long

Matthew A. Romano

Paul C. Tang

Bo Yang

Thomas L. Chenevert 


\title{
Water-fat magnetic resonance imaging quantifies relative proportions of brown and white adipose tissues: ex-vivo experiments
}

\author{
Jadranka Stojanovska, ${ }^{\mathrm{a}, \star}$ Carey N. Lumeng, ${ }^{\mathrm{b}}$ Cameron Griffin, ${ }^{\mathrm{c}}$ Diego Hernando, ${ }^{\mathrm{d}}$ Udo Hoffmann, ${ }^{\mathrm{e}}$ \\ Jonathan W. Haft, ${ }^{f}$ Karen M. Kim, ${ }^{f}$ Charles F. Burant, ${ }^{9}$ Kanakadurga Singer, ${ }^{\text {h }}$ Alex Tsodikov, ${ }^{i}$ \\ Benjamin D. Long, ${ }^{\mathrm{j}}$ Matthew A. Romano, ${ }^{\mathrm{k}}$ Paul C. Tang, ${ }^{\mathrm{k}}$ Bo Yang, ${ }^{\mathrm{k}}$ and Thomas L. Chenevert' \\ aichigan Medicine, Division of Cardiothoracic Radiology, Department of Radiology, Ann Arbor, Michigan, United States \\ ${ }^{b}$ Michigan Medicine, Department of Pediatrics and Molecular Physiology, Ann Arbor, Michigan, United States \\ 'Michigan Medicine, Division of Pediatric Endocrinology, Ann Arbor, Michigan, United States \\ 'University of Wisconsin, Wisconsin Institutes for Medical Research, Medical Physics Department, Madison, Wisconsin, United States \\ eMassachusetts General Hospital, Department of Radiology, Boston, Massachusetts, United States \\ ${ }^{\mathrm{f}}$ Michigan Medicine, Frankel Cardiovascular Center, Department of Cardiac Surgery, Ann Arbor, Michigan, United States \\ IUniversity of Michigan, Ann Arbor, Michigan, United States \\ hMichigan Medicine, Division of Pediatric Endocrinology, Department of Pediatrics and Communicable Diseases, Ann Arbor, Michigan, \\ United States \\ iSchool of Public Health, Ann Arbor, Michigan, United States \\ 'University of Michigan Medical School, Cardiovascular Center, Ann Arbor, Michigan, United States \\ kMichigan Medicine, Cardiovascular Center, Ann Arbor, Michigan, United States \\ 'Michigan Medicine, Department of Radiology-MRI, Ann Arbor, Michigan, United States
}

\begin{abstract}
Quantifying the amount of brown adipose tissue (BAT) within white adipose tissue (WAT) in human depots may serve as a target to combat obesity. We aimed to quantify proton density fat fraction (PDFF) of BAT and WAT in relatively pure and in mixed preparation using water-fat imaging. Three ex-vivo experiments were performed at $3 \mathrm{~T}$ using excised interscapular BAT and inguinal/subcutaneous WAT from mice. The first two experiments consisted of BAT and WAT in separate tubes, and the third used mixed preparation with graded quantities of BAT and WAT. To investigate the influence of partial volume on PDFF metrics, low $\left(2.66 \mathrm{~mm}^{3}\right)$ and high spatial resolution $\left(0.55 \mathrm{~mm}^{3}\right.$ acquired voxels) in two orthogonal three-dimensional sections were compared. The low-resolution acquisitions are corrected for $\mathrm{T}^{*}$ and multipeak lipid spectrum, thus considered "quantitative," whereas the high-resolution acquisitions are not corrected but were performed to better spatially segment BAT from WAT zones. As potential BAT metrics, we quantified the average PDFF and the volume of tissue having PDFF $\leq 50 \%$ (VOL PDFF of BAT was $23 \pm 6 \%$ and $21 \pm 7.6 \%$ and the average PDFF of WAT was $76 \pm 7 \%$ and $87 \pm 7 \%$ using high- and low-resolution techniques, respectively. A similar trend with excellent reproducibility in average PDFF of BAT and WAT was observed in the second experiment. In the third experiment over the four acquisitions, the BAT-dominant tube demonstrated lower PDFF (mean \pm SD) of $55 \pm 2 \%$ than WAT-dominant $(69 \pm 4 \%$ ) and WAT-only tubes $(88 \pm 4 \%)$. Estimating VOL $\mathrm{V}_{\mathrm{PDFF} \leq 50 \%}$, the BAT-dominant tube demonstrated higher volume of $0.26 \mathrm{~cm}^{3}$ than WAT-dominant $\left(0.16 \mathrm{~cm}^{3}\right)$ and WAT-only tubes $\left(0.01 \mathrm{~cm}^{3}\right)$. The presence of BAT exhibits a lower PDFF relative to WAT, thus allowing segmentation of low PDFF tissue for quantification of volume representative of BAT. Future studies will determine the clinical relevance of BAT volume within human depots. $\odot$ The Authors. Published by SPIE under a Creative Commons Attribution 3.0 Unported License. Distribution or reproduction of this work in whole or in part requires full attribution of the original publication, including its DOI. [DOI: 10.1117/1.JMI.5.2.024007]
\end{abstract}

Keywords: multiecho Dixon; brown adipose tissue; white adipose tissue; ex-vivo study; T2*.

Paper 18080R received Apr. 14, 2018; accepted for publication Jun. 8, 2018; published online Jun. 29, 2018.

\section{Introduction}

Brown adipose tissue (BAT) generates heat by nonshivering thermogenesis, ${ }^{1}$ facilitates lipid metabolism, and glucose disposal. ${ }^{2}$ These properties have motivated numerous investigators to study the role of $\mathrm{BAT}^{3-10}$ in the development and management of adiposity and its related comorbidities. ${ }^{11-21}$ ${ }^{18}$ F-fluorodeoxyglucose positron emission tomography

*Address all correspondence to: Jadranka Stojanovska, E-mail: jstoanov@ umich.edu computed tomography detects BAT in adults in a thermoactivated state when actively metabolizing glucose ${ }^{22}$ but fails to detect BAT in a thermoneutral state. Recently published data suggest that magnetic resonance (MR), especially multiecho water-fat imaging (WFI), quantifies BAT in its thermoneutral state. $^{4,5,22,23,24-26}$ Based on the chemical shift, it reliably discriminates water from adipose tissue and quantifies lipid content. This technique has been applied in clinical and preclinical studies ${ }^{24-26}$ by quantifying proton density fat fraction (PDFF) as a potential marker of BAT based on the histologic difference between BAT and white adipose tissue (WAT). The capability of WFI to identify BAT in a thermoneutral state and the lack 
of ionizing radiation ${ }^{22}$ set the stage for MR to represent the most appropriate platform to longitudinally quantify BAT in children and young adults. BAT is found in very small depots spread out in different body parts, most abundantly in the interscapular and supraclavicular regions mixed with white adipocytes. ${ }^{4,5}$ Identifying the presence and the amount of BAT in human depots is pivotal and may serve as a target to combat adiposity and its associated diseases. Therefore, the aim of this study is to quantify the average PDFF and the volume of tissue having

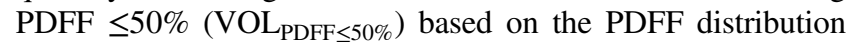
of BAT and WAT on the histogram. We hypothesize that BAT-dominant tissue demonstrates lower PDFF value than WAT-dominant tissue and WAT-only because of the higher content of intracellular water and iron in the mitochondria. ${ }^{5}$ For this aim, we performed ex-vivo WFI experiments on relatively pure and a mixed preparation of excised interscapular BAT and inguinal/subcutaneous WAT from mice. We present results from $e x$ vivo experiments using two WFI techniques for comparison to address the influence of partial volume on PDFF metrics. The first technique uses low-resolution acquisitions that are corrected for $\mathrm{T} 2 *$ and multipeak lipid spectrum, thus considered "quantitative" (Dixon). The second technique uses high-resolution acquisitions that are not corrected for $\mathrm{T} 2 *$ and multipeak lipid spectrum. The high spatial resolution technique was performed to investigate the influence of partial volume effect on PDFF metrics and to better spatially segment BAT from WAT.

\section{Material and Methods}

All mice procedures were approved by the Committee on Use and Care of Animals and were conducted in compliance with the
Institute of Laboratory Animal Research Guide for the Care and Use of Laboratory Animals.

Study design: Preclinical study aimed to quantify the PDFF properties in near homogeneous samples of relatively pure BAT and WAT, as well as inhomogeneous samples containing graded mixtures of BAT with WAT using ex-vivo low- and high-resolution WFI experiments (Fig. 1). This experiment allowed us to obtain PDFF from enriched BAT to quantify BAT volume based on the PDFF histogram.

Excised murine BAT and WAT: Adipose tissue was carefully dissected from 20 young adult (8 to 12 weeks old) wild-type male $\mathrm{C} 57 \mathrm{bl} / 6 \mathrm{~J}$ euthanized mice that were fed a normal diet. The eight euthanized mice that were used in the first ex-vivo experiment were 12 weeks old. The eight euthanized mice that were used in the second ex-vivo experiment were 8 weeks old and the four euthanized mice used in the third experiment were 12 weeks old. The BAT was harvested from interscapular region in $12 \mathrm{C} 57 \mathrm{Bl} / 6$ male mice and the WAT from gonadal and subcutaneous/inguinal regions in 8 mice. The tissues were placed in a 50 cc conical filled with $1 \times$ Dulbecco's phosphate-buffered saline and transported on ice between laboratories.

Histology: Small portion of BAT and WAT at the time of dissection was used for histological confirmation. Light microscopy and immunohistochemistry (IHC) were performed as described elsewhere. ${ }^{27}$ Uncoupled protein-1 (UCP-1) IHC was performed with antibodies at $10 \mu \mathrm{g} / \mathrm{mL}$ (Clone \#536435, R\&D Systems MAB6158) to identify BAT. UCP-1 is a marker of metabolic activity of BAT. ${ }^{3}$

Ex-vivo experiment setup: Three biological ex-vivo imaging experiments were performed. For the first two experiments, the

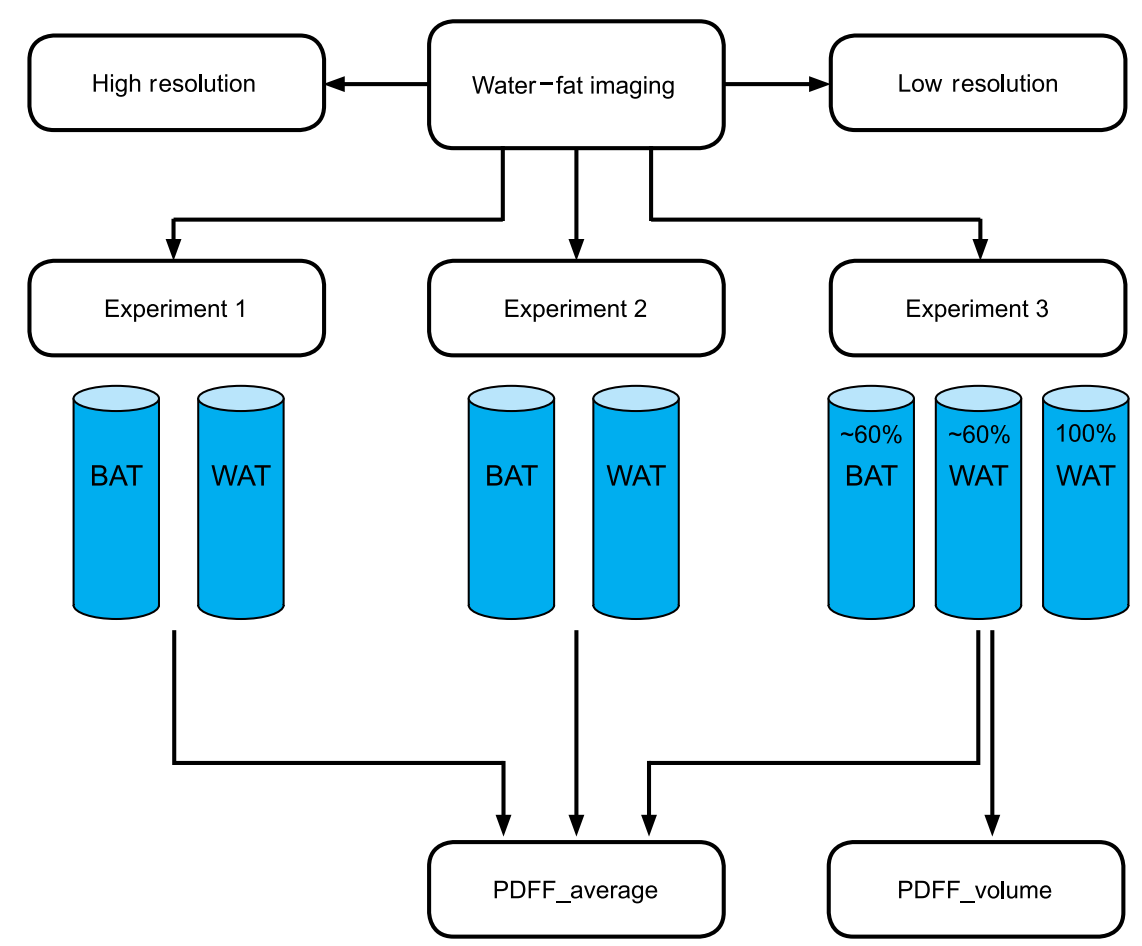

Fig. 1 Flowchart of study design. High and low spatial resolution WFI was performed in three experiments. Experiment 1 and 2 used excised murine BAT and WAT packed in individual tubes. Experiment 3 used a mixed preparation of BAT and perigonadal WAT in the first two tubes and subcutaneous WAT in the third tube. The first tube consisted of $\sim 60 \%$ BAT and the second tube consisted of $\sim 60 \%$ perigonadal WAT. PDFF_average was quantified in all three experiments and PDFF_volume was quantified only in the third experiment. 
excised interscapular BAT and perigonadal WAT were individually packed tightly in two separate microcentrifuge tubes (35$\mathrm{mm}$ long). For the third experiment, a mixture of excised interscapular BAT and perigonadal WAT was densely packed next to each other in two out of the three microcentrifuge tubes $(0.65 \mathrm{cc})$. The first tube consisted of $\sim 60 \%$ interscapular BAT in the mixture of WAT and BAT. The second tube consisted of $\sim 60 \%$ perigonadal WAT in the mixture of WAT and BAT and the third tube consisted of $100 \%$ subcutaneous WAT. We estimated $\sim 60 \%$ composition of tissue by visual inspection while first manually placing the small excised pieces of BAT followed by the pieces of WAT in the vial that was marked at $50 \%$. All tubes were affixed in a $55 \times 75 \times 40 \mathrm{~mm}$ box filled with a mixture of water and gadolinium $(0.5 \mathrm{mmol}$ ProHance, Bracco Diagnostics, New Jersey) to render strong signal by shortening $\mathrm{T} 1$ of water. The samples were set aside to equilibrate to room temperature prior to imaging.

Image acquisition: The ex-vivo experiments were performed on a 3-T MR system (Philips Ingenia, Best, The Netherlands) in an 8-channel wrist coil. To investigate the influence of partial volume on PDFF metrics, two standard WFI techniques (low and high spatial resolution) available on the scanner in two orthogonal three-dimensional (3-D) sections (coronal and sagittal) were used for PDFF measurements. Four total acquisitions, such as low and high spatial resolution, in both sagittal and coronal planes were acquired. The first technique uses multiple single gradient-echo per repetition time (TR) high spatial resolution that is not corrected for $\mathrm{T} 2 *$. The second is 6-gradient-echo quantitative low spatial resolution addressing the $\mathrm{T} 2 *$ bias and multipeak lipid spectrum. ${ }^{28}$ We acquired high spatial resolution WFI to address the influence of partial volume on PDFF metrics and to better spatially segment BAT from WAT. Echo time (TE) was incremented within the high spatial resolution sequence over four sequential acquisitions over 16:54 min $(\mathrm{TE}=3.8,4.8,5.8$, and $6.8 \mathrm{~ms}$ ) for online calculation of water-only, fat-only, and fat-fraction map. This sequence provided higher spatial resolution with the ability to change the acquired voxel size to increase the resolution as opposed to low-resolution quantitative sequence, but the online fat-fraction algorithm did not account for T2* decay. Other technical parameters of the high spatial resolution sequence were: 71 slice $3-\mathrm{D}$ field echo, $\mathrm{TR}=10 \mathrm{~ms}$, flip $=3 \mathrm{deg}, \mathrm{NSA}=6, \mathrm{FOV}=$ $100 \times 60 \mathrm{~mm}$, acquisition voxel $=0.5 \times 0.5 \times 2.2 \mathrm{~mm}^{3}$ (or $0.5 \mathrm{~mm}^{3}$ ), and reconstructed voxel $=0.39 \times 0.39 \times 1.1 \mathrm{~mm}^{3}$. The quantitative sequence acquired six echoes within one acquisition over $7: 82 \mathrm{~min}(\mathrm{TE}=1.5 \mathrm{~ms}+n \times 1.3 \mathrm{~ms}, n=$ $0,1, \ldots, 5)$ for online calculation of water-only, fat-only, fat-fraction, and $\mathrm{T} 2 *$ maps using a multipeak lipid model.
Other technical parameters of the quantitative sequence were: 69 slice 3 -D field echo, $\mathrm{TR}=14 \mathrm{~ms}$, flip $=3 \mathrm{deg}, \mathrm{NSA}=$ $8, \quad \mathrm{FOV}=100 \times 60 \mathrm{~mm}, \quad$ acquisition voxel $=1.1 \times 1.1 \times$ $2.2 \mathrm{~mm}^{3}\left(2.66 \mathrm{~mm}^{3}\right)$, and reconstructed voxel $=0.39 \times 0.39 \times$ $1.1 \mathrm{~mm}^{3}$.

Image postprocessing: MRI DICOM data were converted using in-house MATLAB routines for display and segmentation analysis in 3DSlicer. Volumes of interests (VOI) were placed outlining the adipose tissues. Quantitative evaluation was restricted to four central slices, which showed the lowest levels of artifacts (signal void from air around the plastic lead and at the tip of the tubes) for the first two ex-vivo imaging experiments and to eight center slices for the third ex-vivo imaging experiment. As potential BAT metrics, we quantified the average PDFF and the volume of tissue having PDFF $\leq 50 \%$ $\left(\mathrm{VOL}_{\mathrm{PDFF}} \leq 50 \%\right)$ based on the PDFF histogram. The PDFF statistics for the first two experiments was recorded as VOI average, standard deviation (SD), minimum, and maximum. The PDFF statistics for the third experiment was averaged over the four acquisitions (two orthogonal 3-D sections in high and low spatial resolution) and recorded as mean $\pm \mathrm{SD}$. The volume of relative proportion of BAT having PDFF $\leq 50 \%$ over the four acquisitions in the third experiment was estimated using MATLAB based on the PDFF histogram where we observed the separation point between BAT and WAT. We used fat fraction as an estimation of PDFF.

\section{Results}

Murine BAT and WAT ex-vivo imaging experiments: Murine BAT and WAT were confirmed by histology and IHC. The murine BAT consisted of predominantly small adipocytes positive for UCP-1, while the murine WAT consisted of predominately large adipocytes negative for UCP-1 [Figs. 2(a) and 2(b)]. The results of PDFF measurements for the first two ex-vivo experiments are listed in Table 1 . We found a similar trend of lower PDFF in BAT than in WAT on both quantitative low and high spatial resolution WFI between the first two experiments. The PDFF of WAT was similar between the first two experiments. The second experiment demonstrated excellent reproducibility of the WFI experiment setup. The second experiment showed slightly lower average PDFF of BAT on high spatial resolution image than quantitative low spatial resolution image $(21 \pm 7.6 \%$ and $27 \pm 5 \%)$ (Table 1$)$. By visual inspection, the BAT corresponded with blue color and the WAT with red-yellow color on color-coded fraction maps [Fig. 3(a)]. The third experiment showed a difference in PDFFs between the tubes containing a mixture of BAT and WAT. The BATdominant tube demonstrated average PDFF of $51 \pm 35 \%$ on
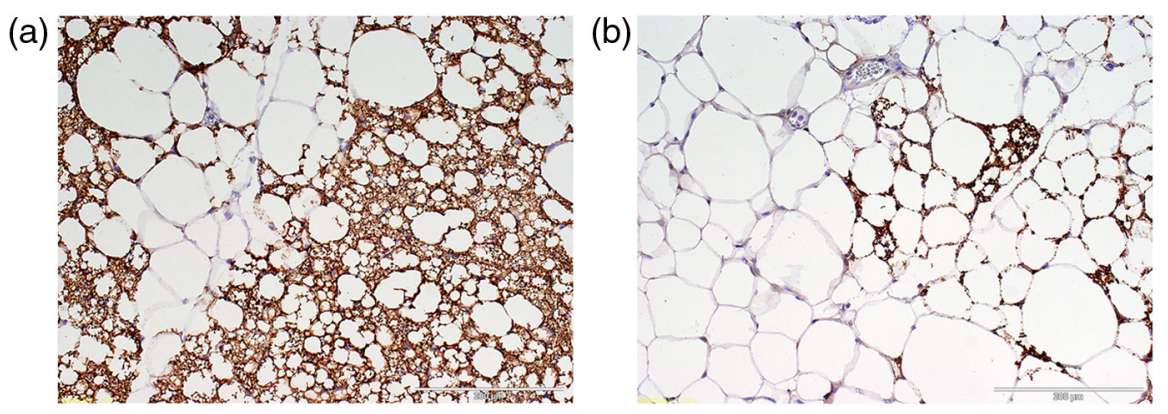

Fig. 2 (a) The histology of the BAT demonstrates small brownish adipocytes positive for UCP-1 and (b) the histology of WAT demonstrates large adipocytes negative for UCP-1. 
Table 1 Water-fat MR imaging distinguishes BAT from WAT.

\begin{tabular}{|c|c|c|c|c|}
\hline \multirow[b]{2}{*}{$\begin{array}{l}\text { PDFF } \\
(\%)\end{array}$} & \multicolumn{2}{|c|}{$\begin{array}{l}\text { Ex-vivo WFI } \\
\text { experiment } 1\end{array}$} & \multicolumn{2}{|c|}{$\begin{array}{l}\text { Ex-vivo WFI } \\
\text { experiment } 2\end{array}$} \\
\hline & $\begin{array}{l}\text { High } \\
\text { resolution }\end{array}$ & $\begin{array}{l}\text { Q Low } \\
\text { resolution }\end{array}$ & $\begin{array}{l}\text { High } \\
\text { resolution }\end{array}$ & $\begin{array}{l}\text { Q Low } \\
\text { resolution }\end{array}$ \\
\hline & $\begin{array}{c}\text { Mean } \pm S D \\
\text { (min to max) }\end{array}$ & $\begin{array}{c}\text { Mean } \pm S D \\
\text { (min to max) }\end{array}$ & $\begin{array}{l}\text { Mean } \pm S D \\
\text { (min to } \max \text { ) }\end{array}$ & $\begin{array}{l}\text { Mean } \pm \text { SD } \\
\text { (min to max) }\end{array}$ \\
\hline BAT & $\begin{array}{c}25 \pm 5 \\
\text { (12 to } 37)\end{array}$ & $\begin{array}{c}23 \pm 6 \\
(13 \text { to } 45)\end{array}$ & $\begin{array}{c}21 \pm 8 \\
\text { (6 to } 39)\end{array}$ & $\begin{array}{c}27 \pm 6 \\
(17 \text { to } 40)\end{array}$ \\
\hline WAT & $\begin{array}{c}87 \pm 7 \\
\text { (66 to } 100)\end{array}$ & $\begin{array}{c}76 \pm 7 \\
(65 \text { to } 93)\end{array}$ & $\begin{array}{c}82 \pm 5 \\
(61 \text { to } 93)\end{array}$ & $\begin{array}{c}73 \pm 6 \\
\text { (56 to } 89)\end{array}$ \\
\hline
\end{tabular}

Note: BAT, brown adipose tissue; WAT, white adipose tissue; $Q$, quantitative; PDFF, proton density fat fraction; and SD, standard deviation.

Note: Ex-vivo experiments from euthanized murine BAT and WAT demonstrating differences in PDFF between BAT and WAT. No difference was seen in PDFF between high spatial resolution and quantitative (Q) low spatial resolution multiecho WFI.

high-resolution images that were lower than the WAT-dominant tube with an average PDFF of $74 \pm 35 \%$ and WAT-only tube with average PDFF of $91 \pm 18 \%$. Similarly, the BAT-dominant tube demonstrated lower PDFF of $52 \pm 29 \%$ on low-resolution images than the WAT-dominant tube with average PDFF of $70 \pm 26 \%$ and WAT-only tube with average PDFF of $84 \pm 12 \%$.
The average PDFF of WAT yielded lower values on lowresolution images compared to high-resolution images. To demonstrate robustness of our third experiment, we calculated mean $\pm \mathrm{SD}$ of average PDFFs over the four acquisitions for each tube. The BAT-dominant tube demonstrated lower mean PDFF of $55 \pm 2 \%$ than WAT-dominant $(69 \pm 4 \%)$ and WATonly tubes $(88 \pm 4 \%)$. Furthermore, in our third ex-vivo experiment, we performed offline histogram analyses to determine the integral volume of relative proportion of BAT within the mixed preparation with WAT up to specified PDFF content over four distinct acquisition series [Figs. 3(a)-3(d)]. Based on the histogram of PDFF averaged over four acquisitions and the BAT distribution within the tissue composition [Fig. 3(c)], we selected a PDFF content up to $50 \%$ for segmentation of the BAT. We then estimated the volume of BAT-like tissue by counting pixels having a PDFF value between $0 \%$ and $50 \%$ (VOL ${ }_{\mathrm{PDFF} \leq 50 \%}$ ) within the total volume of $0.6 \mathrm{~cm}^{3}$. Tube 1 (BAT dominant) demonstrated higher $\mathrm{VOL}_{\mathrm{PDFF} \leq 50 \%}$ of $0.26 \mathrm{~cm}^{3}$ (range 0.23 to $0.28 \mathrm{~cm}^{3}$ ) compared to tube 2 (WAT dominant) with VOL $\mathrm{LDFF} \leq 50 \%$ of $0.16 \mathrm{~cm}^{3}$ (range 0.13 to $0.19 \mathrm{~cm}^{3}$ ) and tube 3 (subcutaneous WAT) with $\mathrm{VOL}_{\mathrm{PDFF} \leq 50 \%}$ of $0.01 \mathrm{~cm}^{3}$ (range 0.01 to $0.02 \mathrm{~cm}^{3}$ ) [Figs. 3(a)-3(d)].

\section{Discussion}

Main findings: Our main findings indicate that average PDFF via multiecho WFI can reliably identify the presence of BAT in thermoneutral state and more importantly can quantify the PDFF volume of relative proportion of BAT mixed with (a)

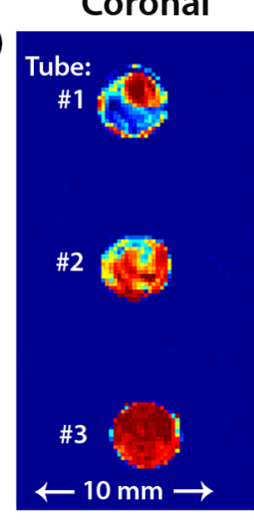

(b)

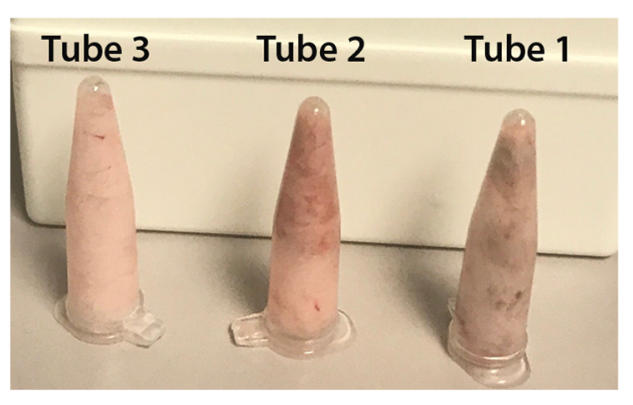

\section{Sagittal}
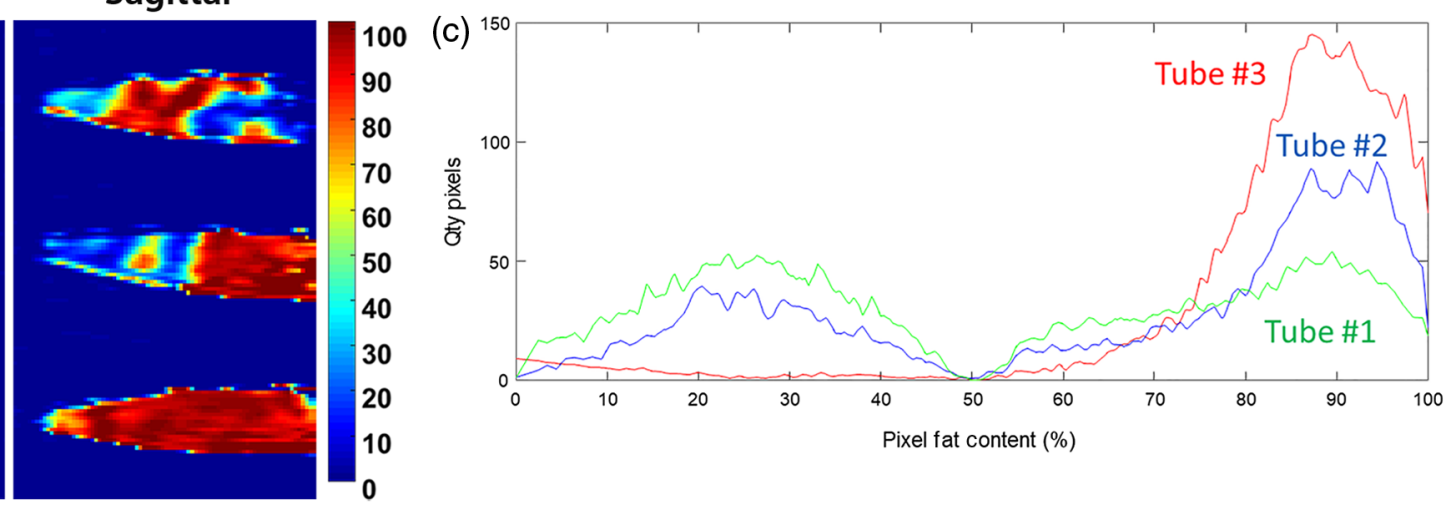

(d)

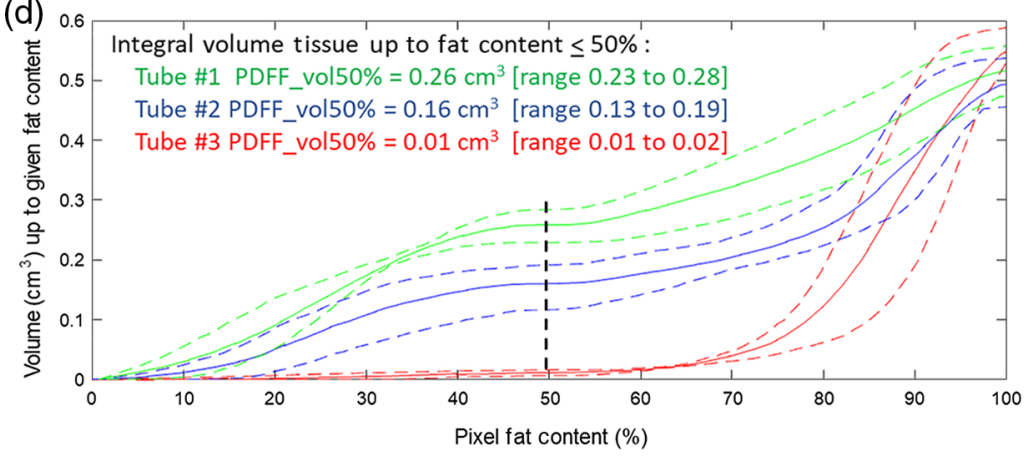

Fig. 3 (a) PDFF map and (b) picture of tubes containing mixtures of white and brown adipose: tube 1 is BAT dominant and shows predominantly blue color, tube 2 is WAT (perigonadal) dominant showing predominantly red color, and tube 3 is WAT (subcutaneous) only showing red color. (c) Histograms of PDFF averaged over four distinct acquisition series. (d) Integral volume of histogram up to specified PDFF content of $50 \%$ (pixel count $\times$ pixel volume) averaged (solid lines), max and min (dash lines) over four distinct acquisition series for tube 1 (green), tube 2 (blue), and tube 3 (red). 
WAT. Excellent reproducibility of our WFI experiment setup was seen in the second experiment. In all three ex-vivo WFI experiments, the presence of BAT demonstrated lower average PDFF than WAT.

Excised mice adipose tissue ex-vivo imaging experiments: Our first two ex-vivo WFI experiments of excised murine BAT and WAT demonstrated lower average PDFF of BAT than WAT using both, high- and low-resolution WFI techniques. The lower average PDFF in brown adipocytes comes from the small intracellular lipid droplets, while the higher PDFF in white adipocytes comes from the large intracellular lipid droplet. ${ }^{5,10,23,24-26}$ It is important to note that in-vivo, brown adipocytes are often found contiguous with white adipocytes..$^{5,7,10,23,24-26}$ Even though PDFF has been shown to reliably distinguish between BAT and WAT, ${ }^{23,24-26}$ in this study, we show the ability of PDFF to quantify the volume of relative proportion of BAT mixed with WAT. Therefore, our third ex-vivo WFI experiment of mixture of murine BAT and WAT yields two quantitative variables: average PDFF to determine the presence and of BAT and $\mathrm{VOL}_{\mathrm{PDFF}}$ to estimate the integral volume of BAT based on the PDFF histogram up to specified PDFF content. This methodology is sufficient if BAT is being identified in large adipose tissue depots, such as supraclavicular depot, or peri-organ adipose tissue, such as peri-renal or epicardial adipose depot. Therefore, quantifying the relative proportion of BAT and WAT within the depot may potentially become an important clinical marker. ${ }^{3,9,29}$ While quantifying PDFF in large adipose depots is feasible, a fundamental limitation of this methodology in small depots is partial volume averaging effects from adjacent organs. ${ }^{23,25}$ Furthermore, voxels at the interface between adipose tissue and adjacent organs may mimic PDFF that is similar to BAT range. ${ }^{23,25}$ A priori knowledge of small depots that would suffer from partial volume effect and validation of BAT with histology should mitigate this limitation. This is especially important if the difference in PDFF between two different depots, such as subcutaneous and supraclavicular, is small ( $82.6 \%$ for supraclavicular versus $90.2 \%$ for subcutaneous). ${ }^{9}$ In the second experiment, we observed slightly lower average PDFF in high spatial resolution than quantitative low-resolution multiecho WFI. This could potentially be explained by the effect of $\mathrm{T} 2 *$ decay that tends to reduce apparent fat fraction on the high spatial resolution image. Of note, we did not observe the same trend in our first experiment. The age difference of the mice between the two experiments may account for slightly different results. We used slightly older mice ( 12 weeks of age) in the first experiment than in the second experiment ( 8 weeks of age). Although 8 to 12 weeks of age is considered young adults for mice, mice that are 12 weeks old may have more white adipocytes in their BAT. However, a similar trend in average PDFF of BAT and WAT was observed between the first two experiments. Our BAT was carefully dissected from the interscapular region of lean mice because the BAT is macroscopically different than the WAT. However, at histology, the BAT contained small amount of white adipocytes. Prakash et al. ${ }^{5}$ have reported slightly increased average PDFF of BAT and slightly decreased average PDFF of WAT in their in-vivo preclinical study than our findings of average PDFF of BAT and WAT. In vivo, it is often difficult to delineate BAT from WAT, and the partial volume effect from field inhomogeneity may alter PDFF values. $\mathrm{Hu}$ et $\mathrm{al}^{23}$ have reported slightly higher but very broad range ( $40 \%$ to $80 \%$ ) of average PDFF mainly for BAT in mice carcasses and excised tissues using methodology similar to our first two ex-vivo imaging experiments. However, this difference is explained by heterogeneous tissue, different scanners, and different preparation techniques.

Prior studies: Hu et al. ${ }^{23}$ and Yokoo et al. ${ }^{30}$ have used a very similar experiment to our first two ex-vivo experiments imaging excised murine BAT and WAT by 6-echo IDEAL (General Electric) algorithm that is similar to our low-resolution multiecho water-fat Dixon (Philips) algorithm. The small difference in the average PDFFs between our studies could be contributed to tissue heterogeneity, different scanners, and tissue preparation techniques. Our tissue samples were obtained from the animal laboratory that was five minutes away from the main hospital where imaging experiments were performed. In addition, we performed third ex-vivo experiment imaging mixture of BAT and WAT and determined the average PDFF and volume of PDFF of relative proportion of BAT within the mixture. Prior studies have calculated fat mass in muscles, ${ }^{31}$ organs, and adipose tissue ${ }^{32}$ using PDFF approach; however, we quantified the relative proportion of BAT mixed with WAT based on a threshold determined by the PDFF histogram. Our third experiment addresses the challenge of extending this technique for clinical application because in humans, pockets of different amount of BAT are found mixed with WAT and rarely as a single contiguous depot.

Limitations: We performed three ex-vivo imaging experiments of euthanized murine BAT and WAT, the first two of which showed excellent reproducibility of this approach, whereas the third addressed partial volume effect of mixtures of tissues. First limitation to translation of this preclinical work is that we imaged excised murine instead of human BAT and WAT. Our third experiment imaging mixture of BAT and WAT brings this concept closer to clinical application because BAT in humans is found mixed with WAT. Second limitation of the translation is the WFI experiment setup. While we cannot translate the same WFI techniques, we will use the same PDFF concept with histogram analysis to quantify the volume of the BAT. The average PDFF of BAT may be lower in live animals than in excised tissue because the difference between room temperature and body temperature influences the fat fraction. ${ }^{33,34}$ Although we found difference in average PDFF between BAT and WAT, further studies in humans with histologic confirmation of WFI findings should address this limitation.

Future direction: Currently, anatomic imaging is used to quantify the total adipose tissue volume in human depots. This approach does not detect the adipose tissue changes in response to cardiometabolic diseases. Segmenting BAT from WAT volume may represent an important estimate of cardiometabolic health. Future studies should determine the clinical relevance of average PDFF and volume PDFF of BAT as an estimate of obesity phenotypes, risk stratification, and guide management of cardiometabolic disease.

\section{Conclusion}

This work investigates the feasibility of quantifying the volume of relative proportion of BAT mixed with WAT. BAT and WAT show different PDFF behavior. BAT demonstrates lower average PDFF than WAT in relatively pure and in mixed preparation with WAT. It also demonstrates a higher volume of BAT in the BATdominant tube compared to WAT-dominant and WAT-only tubes. Multiecho WFI can be used to quantify the presence 
and the volume of relative proportion of BAT within the depot of interest. These metrics will facilitate longitudinal measurements in human studies; however, future research is needed for validation purposes.

\section{Disclosures}

No conflicts of interest, financial or otherwise, are declared by the authors.

\section{Acknowledgments}

The content is solely the responsibility of the authors and does not necessarily represent the official views of the National Institutes of Health. We would like to acknowledge the contribution of Vanessa Allen in providing high-quality figures. Dr. Stojanovska reports a training grant from the National Center for Advancing Translational Sciences (NCATS). Dr. Lumeng, Dr. Burant, Dr. Hernando, and Dr. Singer report grants from the National Institute of Diabetes and Digestive and Kidney Diseases during the conduct of the study. Dr. Hernando also reports grant from the National Institute of Biomedical Imaging and Bioengineering. This research was supported by the training grant from NCATS under the award Grant Nos. F043322 and U052387. This research was conducted in compliance with the Institute of Laboratory Animal Research Guide for the Care and Use of Laboratory Animals and approved by the Committee on Use and Care of Animals.

\section{References}

1. J. Nedergaard, T. Bengtsson, and B. Cannon, "New powers of brown fat: fighting the metabolic syndrome," Cell Metab. 13(3), 238-240 (2011).

2. A. Bartelt et al., "Brown adipose tissue activity controls triglyceride clearance," Nat. Med. 17, 200-205 (2011).

3. A. M. Cypress et al., "Identification and importance of brown adipose tissue in adult humans," N. Engl. J. Med. 360, 1509-1517 (2009).

4. Y. I. Chen et al., "Measurement of human brown adipose tissue volume and activity using anatomic MR imaging and functional MR imaging," J. Nucl. Med. 54(9), 1584-1587 (2013).

5. K. N. B. Prakash et al., "A method for the automatic segmentation of brown adipose tissue," Magn. Reson. Mater. Phys. 29, 287-299 (2016).

6. H. S. Sacks and J. N. Fain, "Human epicardial adipose tissue: a review," Am. Heart J. 153(6), 907-917 (2007)

7. G. Iacobellis, "Local and systemic effects of the multifaceted epicardial adipose tissue depot," Nat. Rev. Endocrinol. 11, 363-371 (2015).

8. H. S. Sacks et al., "Uncoupling protein-1 and related messenger ribonucleic acids in human epicardial and other adipose tissues: epicardial fat functioning as brown fat," J. Clin. Endocrinol. Metab. 94(9), 36113615 (2009).

9. B. T. Franssens et al., "Relation between brown adipose tissue and measures of obesity and metabolic dysfunction in patients with cardiovascular disease," J. Magn. Reson. Imaging 46(2), 497-504 (2017).

10. H. S. Sacks et al., "Adult epicardial fat exhibits beige features," J. Clin. Endocrinol. Metab. 98(9), E1448-E1455 (2013).

11. R. B. Ervin, "Prevalence of metabolic syndrome among adults 20 years of age and over, by sex, age, race and ethnicity, and body mass index: United States, 2003-2006," Natl. Health Stat. Report 5(13), 1-7 (2009).

12. A. A. Mahabadi et al., "Association of epicardial adipose tissue with progression of coronary artery calcification is more pronounced in the early phase of atherosclerosis," JACC Cardiovasc. Imaging 7(9), 909-916 (2014).

13. K. F. Adams et al., "Overweight, obesity, and mortality in a large prospective cohort of persons 50 to 71 years old," New Engl. J. Med. 355, 763-778 (2006).

14. H. M. Lakka et al., "The metabolic syndrome and total and cardiovascular disease mortality in middle-aged men," J. Am. Med. Assoc. 288(21), 2709-2716 (2002).
15. S. Malik et al., "Impact of the metabolic syndrome on the mortality from coronary heart disease, cardiovascular disease, and all causes in United States adults," Circulation 110(10), 1245-1250 (2004).

16. A. Mente et al., "Metabolic syndrome and risk of acute myocardial infarction a case-control study of 26, 903 subjects from 52 countries," J. Am. Coll. Cardiol. 55(21), 2390-2398 (2010).

17. S. Mottillo et al., "The metabolic syndrome and cardiovascular risk a systematic review and meta-analysis," J. Am. Coll. Cardiol. 56(14), 1113-1132 (2010).

18. A. A. Mahabadi et al., "Association of pericardial fat, intrathoracic fat, and visceral abdominal fat with cardiovascular disease burden: the Framingham Heart Study," Eur. Heart J. 30(7), 850-856 (2009).

19. J. Stojanovska et al., "Increased epicardial fat volume is independently associated with presence of atrial fibrillation, atrial fibrillation chronicity and radiofrequency ablation outcome," Eur. Radiol. 25(8), 22982309 (2015).

20. B. Long et al., "Increased epicardial fat volume is independently associated with the presence and severity of systemic sclerosis," Acad. Radiol. 24, 1473-1481 (2017).

21. A. Whittle and A. Vidal-Puig, "When BAT is lacking, WAT steps up," Cell Res. 23(7), 868-869 (2013).

22. D. Franz et al., "Discrimination between brown and white adipose tissue using a 2-point Dixon water-fat separation method in simultaneous PET/MRI," J. Nucl. Med. 56(11), 1742-1747 (2015).

23. H. H. Hu et al., "Identification of brown adipose tissue in mice with fatwater IDEAL-MRI," J. Magn. Reson. Imaging 31(5), 1195-1202 (2010).

24. S. C. N. Hui et al., "Quantification of brown and white adipose tissue based on Gaussian mixture model using water-fat and T2* MRI in adolescents," J. Magn. Reson. Imaging 46, 758-768 (2017).

25. H. H. Hu et al., "Comparison of brown and white adipose tissues in infants and children with chemical-shift-encoded water-fat MRI," J. Magn. Reson. Imaging 38, 885-896 (2013).

26. H. H. Hu et al., "Characterization of human brown adipose tissue by chemical-shift water-fat MRI," Am. J. Roentgenol. 200, 177-183 (2013).

27. C. N. Lumeng, J. L. Bodzin, and A. R. Saltiel, "Obesity induces a phenotypic switch in adipose tissue macrophage polarization," J. Clin. Invest. 117(1), 175-184 (2007).

28. C. D. Hines et al., "T1 independent, T2* corrected MRI with accurate spectral modeling for quantification of fat: validation in a fat-waterSPIO phantom,” J. Magn. Reson. Imaging 30(5), 1215-1222 (2009).

29. C. M. Phillips, "Metabolically healthy obesity: definitions, determinants and clinical implications," Rev. Endocr. Metab. Disord. 14, 219-227 (2013).

30. T. Yokoo et al., "Linearity, bias, and precision of hepatic proton density fat fraction measurements by using MR imaging: a meta-analysis," Radiology 286(20), 486-498 (2018).

31. L. C. Doro et al., "Validation of an adapted MRI pulse sequence for quantification of fatty infiltration in muscle," Magn. Reson. Imaging 27(6), 823-827 (2009).

32. H. H. Hu et al., "Quantification of absolute fat mass by magnetic resonance imaging: a validation study against chemical analysis," Int. J. Body Compos. Res. 9(3), 111-122 (2011).

33. D. Hernando et al., "On the confounding effect of temperature on chemical shift-encoded fat quantification," Magn. Reson. Med. 72(2), 464-470 (2014).

34. D. Hernando et al., "R2* estimation using "in-phase" echos in the presence of fat: the effects of complex spectrum of fat," J. Magn. Reson. Imaging 37(3), 717-726 (2013).

Jadranka Stojanovska is an assistant professor of radiology on an instructional track, a board-certified diagnostic radiologist, and a fellowship trained cardiothoracic radiologist. Following her medical training and residency in radiology, she took a traditional fellowship in cardiothoracic radiology at the University of Michigan. She obtained her master of science degree in clinical research and statistical design from the University of Michigan School of Public Health. Her research focuses on translational and outcomes research using quantitative imaging in risk stratification and therapeutic efficacy assessment of cardiovascular diseases, metabolic syndrome, and obesity.

Biographies for the other authors are not available. 